\title{
Antimicrobial Profiles of Selected Gram-Negative Bacteria Recoverable from Sewage and Sludge from Juja and Kibera Informal Settlements of the Larger Nairobi Metropolis
}

\author{
John Maina ${ }^{1,2 *}$, Perpetual Ndung' ${ }^{1}{ }^{1}$, Anne Muigai ${ }^{1}$, Helen Onyango ${ }^{1}$, Joel K. Mukaya ${ }^{1}$, \\ Susan Wambui ${ }^{2}$, Terry Judah ${ }^{2}$, Joyce Kinyua ${ }^{1}$, Joystella Muriuki ${ }^{1}$, Lynne Chesenge ${ }^{1}$, Lydia Kisoo' ${ }^{1}$, \\ Rebecca Thuku1', Boniface Wachira1 ${ }^{1}$, Vincent Bett ${ }^{2}$, Thomas Gachuki ${ }^{1}$, John Kiiru ${ }^{2 *}$
}

${ }^{1}$ Jomo Kenyatta University of Agriculture and Technology, Juja, Kiambu County, Central Kenya, Kenya

${ }^{2}$ Center for Microbiology Research, Kenya Medical Research, Nairobi, Kenya

Email: ${ }^{*}$ johnmaina.jnm@gmail.com

How to cite this paper: Maina, J., Ndung'u, P., Muigai, A., Onyango, H., Mukaya, J.K., Wambui, S., Judah, T., Kinyua, J., Muriuki, J., Chesenge, L., Kisoo, L., Thuku, R., Wachira, B., Bett, V., Gachuki, T. and Kiiru, J. (2019) Antimicrobial Profiles of Selected Gram-Negative Bacteria Recoverable from Sewage and Sludge from Juja and Kibera Informal Settlements of the Larger Nairobi Metropolis. Advances in Microbiology, 9, 507-524.

https://doi.org/10.4236/aim.2019.96031

Received: May 2, 2019

Accepted: June 15, 2019

Published: June 18, 2019

Copyright $\odot 2019$ by author(s) and Scientific Research Publishing Inc. This work is licensed under the Creative Commons Attribution International License (CC BY 4.0).

http://creativecommons.org/licenses/by/4.0/ (c) (i) Open Access

\begin{abstract}
Africa has experienced rapid urban migration in the past two decades. New informal settlements continue to emerge and expand but the sanitation provision of facilities has not improved at the same pace and this poses a serious health concern to the public especially the urban poor. Open sewage systems and sludge-clogged drainage systems as well as soil contaminated with industrial and domestic wastes are possible sources of germs that probably cause clinical infections and epidemics. In this cross-sectional study, we recorded diverse genera of Gram-negative non-fastidious bacteria that included; Escherichia coli (23\%), Klebsiella spp (21\%), Enterobacter spp (19\%), Citrobacter spp (10\%), Pseudomonas aeruginosa (8\%), Proteus spp (7\%), Salmonella (3\%), Yersinia spp (3\%), Shigella spp (2\%), Morganella morganii (2\%), Edwardisella spp (1\%), Hafnia spp (1\%), Serratia marcesence (0.5\%), and Acinetobacter baumannii (0.5\%). Most of these isolates were resistant to ampicillin while imipenem and ciprofloxacin were the most effective antimicrobial agents. Resistance combination towards ampicillin, trimethoprim, sulfamethoxazole and streptomycin was also noted in recovered isolates (16\%). An overall high antimicrobial resistance was recorded among isolates from slum as compared to those recovered from Juja, a middle-class settlement located at the edge of Nairobi metropolis. The prevalence of isolates with a combined resistance to $3^{\text {rd }}$ generation cephalosporins (cefotaxime, ceftriaxone and ceftazidime), gentamicin and ciprofloxacin was the highest among $P$. aeruginosa
\end{abstract}


isolates (13\%) but none of the Yersinia species and Edwardisella tarda exhibited this resistance. Carriage of bla ria species followed by bla $a_{\text {CTX-M }}(20 \%)$, bla $a_{S H V}(18 \%)$ while bla $a_{O X A}(17 \%)$ was the least common. The phylogeny analysis revealed significant genetic similarity among strains belonging to E. coli, K. pneumoniae, E. agglomerans and $P$. mirabilis strains but less relatedness was noted among strains belonging to $C$. freundii. Further analysis showed possible clonal expansion of $E$. agglomerans and $K$. pneumoniae within the environmental ecosystems.

\section{Keywords}

Antimicrobial Resistance, Multiple-Drug Resistance (MDR), Extended Spectrum $\beta$-Lactamases (ESBL), Enterobacteriaceae, Sewage and Sludge

\section{Introduction}

The emergence and spread of antimicrobial resistance have become a global concern and affect our ability to combat severe infections. It is estimated that nearly $1 \mathrm{M}$ deaths annually occur across the globe as a result of treatment failure due to antimicrobial resistance [1]. Notably, in resource-poor countries, especially those in Sub-Sahara Africa, cases of morbidity and mortality due to treatment failure are on the rise. The increase in infections caused by Gram-negative bacteria has also led to a surge in usage and over-reliance on antimicrobials for human and animal infection treatment and prophylaxis.

As a result of over-use and misuse of antibiotics in human and animal health, domestic waste is likely to be highly contaminated with fecal materials which in turn have bacteria that are resistant to multiple antimicrobials due to their possible clinical origin [2]. Previous studies have documented sludge and sewage effluents as major sources of antimicrobial resistant bacteria strains in the environmental compartment [3] [4].

Antimicrobial resistance in Gram-negative bacteria is on the rise which is partially attributed to the ease of spread and acquisition of resistance genes especially amongst the members of the family Enterobacteriaceae. Horizontal transfer of these resistance genes has largely been attributed to plasmid-borne integrons which are able to assemble resistance genes in cassettes [5]. Multiple-drug resistance (MDR) strains in sewage, sludge or soil can easily get into water bodies such as river and municipal water supply lines and end up as clinical strains responsible for various infections in humans and animals. This is even more plausible in informal settlements where water supply lines often pass through open sewers and where many illegal water connections happen. In such settings, most of street food-vending points are often situated next to open sewer and hand hygiene is hardly observed further increasing risk of acquisition and spread of MDR-strains. Therefore, foods sold in such environments can cause food borne infections and outbreaks. Some antimicrobial agents such as tetracycline 
and quinolone have a long shelf-life in environmental compartments while others such as sulfonamides are water soluble and their persistence may create selection pressure that leads to emergence of resistant clones [6] [7]. Contamination and long-term exposure of soil to antimicrobial residuals due to livestock rearing and agricultural practices may therefore lead to resistance build-up and positively select for highly resistant strains [8].

In Kenya, like in most sub-Saharan countries, the role played by sewage, sludge and fecal contaminated soils as sources for isolates with MDR strains has not been investigated. This is even more important in informal settlements characterized by poor sanitation, limited access to clean water and over-congestion [9]. Such data is particularly important because it may find important applications in outbreaks management and in policy formulations to curb spread of AMR and for identification of hotspots that play a role as flush-points for outbreaks associated with MDR strains.

In this cross-sectional study, we determined the diversity of non-fastidious Gram-negative bacteria and their associated antimicrobial resistance patterns to common antimicrobials and the phylogenic relatedness of strains from different sites. The current study was conducted in Kibera informal settlement in Nairobi which is the second largest slum in Africa continent and in Juja town, a middle-class settlement at the edge of Nairobi metropolis.

\section{Materials and Methodology}

\subsection{Study Design and Sample Collection}

A convenient random sampling design was used in this cross-sectional study to obtain soil, sludge and sewage samples from sites near food vending point in Kibera informal settlements and Juja town, Figure 1 and Figure 2. A purposeful 100 samples each of sewage, sludge and soil were obtained across 13 villages of

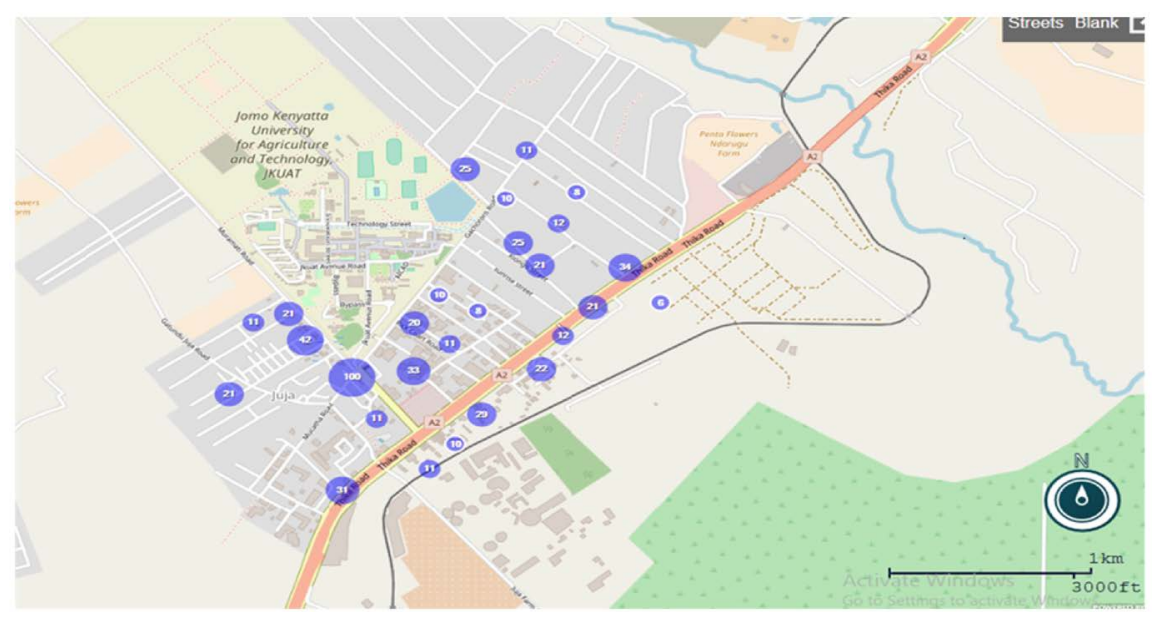

Figure 1. Distribution pattern of multiple drugs resistance Gram negative bacteria isolates from Juja metropolis. The spot map show the distribution pattern of Enterobacteriaceae isolates that were resistant to more than 3 antimicrobial agents belonging to different classes. 


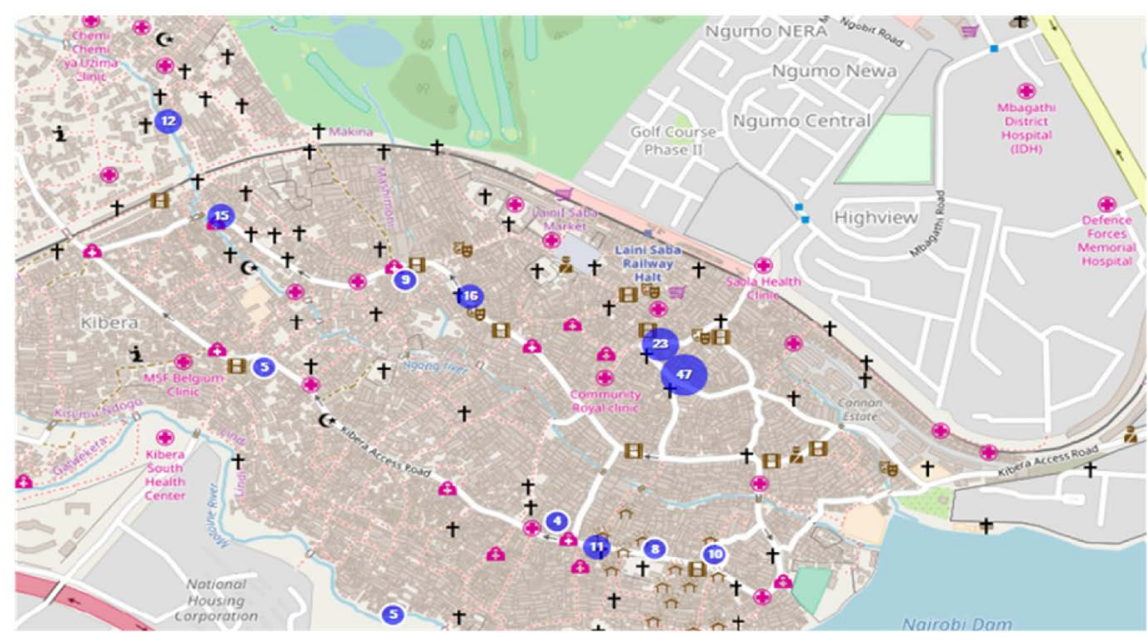

Figure 2. Distribution pattern of multiple drugs resistance Gram negative bacteria isolates from Kibera slums. The spot map shows the distribution pattern of Enterobacteriaceae isolates that were resistant to more than 3 antimicrobial agents belonging to different classes.

Kibera slum between July to December 2017. During that period, a similar set of samples were also obtained in Juja town. Approximately $30 \mathrm{ml}$ of sewage and sludge sample was obtained by holding universal bottle at the base and plunging it below the surface of flowing sewer. Approximately $30 \mathrm{~g}$ of soil sample was also collected near the point of sewage and sludge collection site. The samples were then transported to laboratory within 2 hours for processing and culture.

\subsection{Sample Processing}

In order to isolates non-fastidious Gram-negative bacteria especially those belonging to family Enterobacteriaceae, approximately $1 \mathrm{~mL}$ of sewage or sludge sample was inoculated in $9 \mathrm{ml}$ of buffered peptone water and alkaline peptone water, shaken for $1 \mathrm{hr}$ in order to resuscitate any injured cells before plating directly onto oxoid ${ }^{\text {tm }}$ MacConkey and Eosin Methylene Blue Agar (EMBA) and blood agar (BA) plates. From each plate, three distinct colonies of similar morphology were purified on EMBA and on tryptone soy agar (TSA). Pure isolates were identified through Gram-staining and a series of biochemical tests that included; triple sugar iron, lysine-indole motility, citrate utilization, methyl red voges proskauer test and urease test as described in the past [10].

\subsection{Antimicrobial Susceptibility Testing}

Antimicrobial susceptibility profiles were performed using the disk diffusion method on Mueller Hinton medium. The antimicrobial panel used consisted of ampicillin (AMP, $10 \mu \mathrm{g}$ ), amoxicillin/clavulanic acid (AMC, 20/10 $\mu \mathrm{g}$ ), cefoxitin (FOX, $30 \mu \mathrm{g}$ ), ceftriaxone (CRO, $30 \mu \mathrm{g}$ ), cefotaxime (CTX, $30 \mu \mathrm{g}$ ), ceftazidime (CAZ, $30 \mu \mathrm{g}$ ), cefepime (FEP, $30 \mu \mathrm{g}$ ), aztreonam (ATM, $30 \mu \mathrm{g}$ ), ciprofloxacin (CIP, $5 \mu \mathrm{g}$ ), nalidixic acid (NA, $30 \mu \mathrm{g})$, gentamicin (CN, $10 \mu \mathrm{g})$, streptomycin (S, $10 \mu \mathrm{g}$ ), imipenem (IPM, $10 \mu \mathrm{g}$ ), sulfamethoxazole (RL, $200 \mu \mathrm{g}$ ) and trimetho- 
prim (W, $5.2 \mu \mathrm{g}$ ). E. coli ATCC 25922 reference strains was used for ensuring media quality and disc potency. Interpretation of antimicrobial susceptibility zones was done using the CLSI 2017 guidelines. Chi-square test was used for statistic analysis where a value of 0.05 or less was considered as indication of significant difference between test variables.

\subsection{PCR Detection of $\beta$-Lactamase Genes}

Extraction of DNA was done using the boiling method. This process entailed emulsifying bacterial colonies in $1000 \mu \mathrm{l}$ molecular grade water. Cell lyses was then done by boiling the preparation at $95^{\circ} \mathrm{C}$ on a thermal block for $10-15 \mathrm{~min}$. Separation was the done through centrifugation at 14,000 rpm for 5 minutes. The supernatant containing DNA was then stored at $-20^{\circ} \mathrm{C}$. Detection of genes that are frequently associated with resistance to important classes of B-lactams such as bla $a_{\text {TEM }}, b a_{\text {СTX-M }}, b l a s \mathrm{sV}, b l a_{\mathrm{OXA}}$ and class 1 integron was done using published primers, Table 1 [11] [12] [13]. The final volume in each PCR tube was 25 $\mu \mathrm{l}$ which included $10 \mu \mathrm{l}$ of Qiagen master mix, $1 \mu$ butane, 2 ul DNA, $10 \mu \mathrm{l}$ PCR water and $2 \mu \mathrm{l}$ forwards and reverse primer. Amplified PCR products were separated in $1.5 \%$ gel and banding patterns visualized under UV gel imager.

\subsection{Finger Printing of Recovered Bacterial Isolates}

Phylogenetic relatedness was determined using the (GTG) $)_{5}$-PCR method using published strategies and primers indicated in Table 1. (13) PCR amplification of target DNA sequence was done at $40^{\circ} \mathrm{C}$ annealing temperature. Amplified products were separated by running in $1 \%$ agarose gel for I hour. Visualization of banding patterns was done using a Gelmax ${ }^{\circledast}$ UV imager. Banding patterns were analyzed using bionumerics Gelcompar 2 software version 6.6 with the cluster

Table 1. PCR amplification primers used.

\begin{tabular}{|c|c|c|c|c|c|}
\hline Target gene & Primer name & Primer sequence & Annealing $\mathrm{T}\left({ }^{\circ} \mathrm{C}\right)$ & Product size & Reference \\
\hline \multirow{2}{*}{ blaTEM } & TEM-F & 5'-GCGGAACCCCTATTTG-3' & 50 & 964 bp & \\
\hline & & 5'-TCTAAAGTATATATGAGTAAACTTGGTCTGAC-3' & & & 12 \\
\hline \multirow{2}{*}{ blaCTX-M } & CTX-M-F & 5'-ATGTGCAGYACCAGTAARGTKATGGC-3' & 60 & $593 \mathrm{bp}$ & 12 \\
\hline & CTX-M-R & 5'-TGGGTRAARTARGTSACCAGAAYCAGCGG-3' & & & \\
\hline \multirow{2}{*}{ blaSHV } & SHV-F & 5'-TTCGCCTGTGTATTATCTCCCTG-3' & 50 & 854 bp & 12 \\
\hline & SHV-R & 5'-TTAGCGTTGCCAGTGYTCG-3' & & & \\
\hline \multirow{2}{*}{ blaOXA } & OXA-IF & 5'-ATGAAAAACACAATACATATCAACTTCGC-3' & 62 & 820 bp & 12 \\
\hline & 0XA-1R & 5’-GTGTGTTTAGAATGGTGATCGCATT-3' & & & \\
\hline \multirow{2}{*}{ Int 1} & intM1_D & 5'-GAAAGGTCTGGTCATACATG-3' & 50 & $500 \mathrm{bp}$ & 11 \\
\hline & intM1_U & 5'ACGAGCGCAAGGTTTCGGT-3" & & & \\
\hline (GTG)5 & (GTG)5 & 5’-GTGGTGGTGGTGGTG-3' & 50 & variable & 13 \\
\hline
\end{tabular}

Key: bla: $\beta$-lactamase gene, bp: molecular weight in base pair. 
analysis done using the dice method based on UPGMA arithmetic mean. Randomly selected bacteria species from six most prevalent genera were analyzed for genetic relatedness. A correlation of $\geq 80 \%$ among bacterial species was considered as strong evidence of genetic relatedness among isolates as previously described [14].

\subsection{Ethical Approval}

Ethical approval prior to the study commence was obtained from scientific ethical review unit, Kenya medical research institute (SERU, KEMRI), approval number KEMRI/SERU/CMR/P00055/3514.

\section{Results}

\subsection{Bacterial Isolates}

A total of 348 Gram-negative isolates were obtained from sewage, 364 from sludge and 211 from soil, Table 2. A total of 14 non-fastidious bacteria genera were identified from these samples obtained from the slum (Kibera) and from middle class settings (Juja). Isolates obtained in this study were categorized in 4 groups; indicator organisms for AMR trends in human, animal and the environment [Escherichia (23\%) and Klebsiella (21\%)], genera of known Enteropathogens species [Salmonella (3\%) and Shigella (2\%)], genera associated with high intrinsic antmicrobial resistances $[A$. baumannii $(0.5 \%), P$. aeruginosa (8\%), M. morganii (2\%), Sr. marcesence (0.5\%)] and other genera that are not significantly associated with diseases but significant as opportunistic infection pathogens such as [Citrobacter spp (10\%), Enterobacter spp (19\%), Edwardisella spp (1\%), Proteus spp (7\%), Yersinia spp (3\%) and Hafnia spp (1\%)].

Table 2. Bacterial diversity from sewage and sludge samples.

\begin{tabular}{|c|c|c|c|c|c|c|c|c|c|c|c|c|c|c|}
\hline \multicolumn{15}{|c|}{ Gram negative bacteria diversity in environmental samples } \\
\hline & 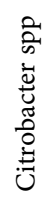 & 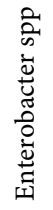 & 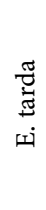 & $\begin{array}{c}\ddot{\overline{0}} \\
\dot{0} \\
\text { ن }\end{array}$ & $\begin{array}{l}\overline{\vec{\nu}} \\
\vec{\sigma} \\
\vec{\Psi}\end{array}$ & 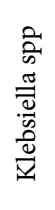 & 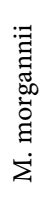 & 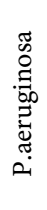 & 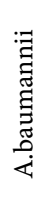 & 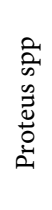 & 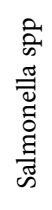 & 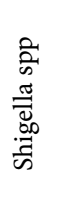 & 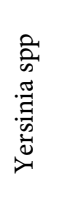 & 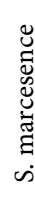 \\
\hline \multicolumn{15}{|l|}{ Sewage } \\
\hline Juja $\mathrm{n}=139$ & 17 & 28 & 0 & 38 & 0 & 31 & 0 & 10 & 0 & 10 & 0 & 0 & 5 & 0 \\
\hline Kibera $\mathrm{n}=209$ & 10 & 31 & 10 & 43 & 10 & 33 & 5 & 13 & 5 & 14 & 15 & 10 & 10 & 0 \\
\hline \multicolumn{15}{|l|}{ Sludge } \\
\hline Juja $\mathrm{n}=153$ & 22 & 33 & 0 & 39 & 0 & 35 & 0 & 12 & 0 & 12 & 0 & 0 & 0 & 0 \\
\hline Kibera $\mathrm{n}=211$ & 25 & 42 & 0 & 32 & 0 & 41 & 10 & 18 & 0 & 15 & 12 & 6 & 10 & 0 \\
\hline \multicolumn{15}{|l|}{ Soil } \\
\hline Juja $\mathrm{n}=75$ & 9 & 12 & 0 & 25 & 0 & 22 & 0 & 7 & 0 & 0 & 0 & 0 & 0 & 0 \\
\hline Kibera $n=136$ & 10 & 30 & 0 & 36 & 0 & 30 & 0 & 10 & 0 & 15 & 0 & 0 & 0 & 5 \\
\hline
\end{tabular}

Lctn: Location, spp: species, n: total number, $E$. tarda: Edwardisella tarda, $E$. coli: Escherichia coli, $P$. aeruginosa: Pseudomonas aeruginosa, $S$. marcesence: Serratia marcesence. 


\subsection{Spatial Difference in Antimicrobial Resistance Profiles}

Isolates from slum sludge were overly more resistant to any tested antimicrobial agents. High resistance prevalence was recorded towards ampicillin (AMP 57\%), trimethoprim (W 56\%) and sulfamethoxazole (RL 55\%) among sludge isolates recovered from the slum, Table 3 . Soil isolates from Juja middle class setting were the least resistance to any tested antimicrobial agent. An overall high antimicrobial resistance was recorded in isolates from slum as compared to those recovered from Juja, a town located at the edge of Nairobi metropolis. Resistance towards cephalosporins was relatively higher among slum isolates with highest resistance values recorded against ceftriaxone (CRO 14\%), cefotaxime (CTX $12 \%$ ) and ceftazidime (CAZ 11\%) respectively. Further analysis showed significant difference in cephalosporin resistance e.g. against ceftazidime resistance between the 2 study sites [OR: 0.31, CI 0.59, P: 0.00002)]. Isolates recovered from the slums area (Kibera) were more resistant to ciprofloxacin than those from obtained from the middle-class area where a significant difference was also noted (OR: 2.13, CI: 3.69, P: 0.006). Slum isolates were more resistance to aminoglycosides, with no significant difference been recorded [gentamicin (OR 1.26, CI 1.93, P: 0.28)]. Most isolates were resistant to ampicillin while imipenem and ciprofloxacin were the most effective antimicrobial agents.

\subsection{Antimicrobial Resistance Patterns Based on Species}

Among the 14 bacteria genera reported in this study, $P$. aeruginosa isolates were the most resistant and on average were resistant to at least 9 of the tested antimicrobial agents, Table 4 . This species also had the highest prevalence of isolates $(13 \%)$ with a combined resistance to $3^{\text {rd }}$ generation cephalosporins (ceftriaxone $22 \%$, cefotaxime $20 \%$, and ceftazidime $15 \%$ ), advanced classes of aminoglycosides (gentamicin 20\%) and otherwise potent classes of quinolones (ciprofloxacin

Table 3. Antimicrobial resistance patterns of all bacterial isolates recovered from Juja and Kibera.

\begin{tabular}{|c|c|c|c|c|c|c|c|c|c|c|c|c|c|c|c|c|}
\hline & \multicolumn{16}{|c|}{ Antimicrobial resistance $\%$} \\
\hline & $\mathrm{n}$ & AMP & CAZ & CTX & CRO & FEP & ATM & AMC & FOX & CN & $S$ & CIP & NA & IPM & RL & $\mathrm{W}$ \\
\hline \multicolumn{17}{|l|}{ Sewage } \\
\hline Juja & 139 & 50 & 8 & 10 & 11 & 5 & 14 & 11 & 22 & 10 & 19 & 5 & 18 & 0 & 42 & 39 \\
\hline Kibera & 209 & 53 & 9 & 11 & 13 & 7 & 15 & 17 & 24 & 12 & 23 & 10 & 21 & 1 & 39 & 43 \\
\hline \multicolumn{17}{|l|}{ Sludge } \\
\hline Juja & 153 & 55 & 10 & 11 & 12 & 6 & 15 & 18 & 26 & 12 & 20 & 6 & 23 & 0 & 46 & 48 \\
\hline Kibera & 211 & 57 & 11 & 12 & 14 & 7 & 19 & 19 & 28 & 14 & 27 & 12 & 26 & 0 & 55 & 56 \\
\hline \multicolumn{17}{|l|}{ Soil } \\
\hline Juja & 75 & 33 & 5 & 8 & 9 & 4 & 13 & 15 & 16 & 6 & 20 & 3 & 13 & 0 & 33 & 35 \\
\hline Kibera & 136 & 40 & 7 & 9 & 10 & 6 & 10 & 15 & 15 & 10 & 22 & 7 & 22 & 0 & 40 & 44 \\
\hline
\end{tabular}

AMP: Ampicillin, CTX: Cefotaxime, CRO: Ceftriaxone, FEP: Cefepime, ATM: Aztreonam, FOX: Cefoxitin, AMC: Amoxicillin-clavulanic acid, CN: Gentamicin, S: Streptomycin, CIP: Ciprofloxacin, NA: Nalidixic acid, IPM: Imipenem, RL: Sulfamethoxazole, W: Trimethoprim, $n=$ total number bacteria isolates. 
Table 4. Antimicrobial resistance profiles of sewage, sludge and soil samples.

\begin{tabular}{|c|c|c|c|c|c|c|c|c|c|c|c|c|c|c|c|c|c|}
\hline & \multicolumn{17}{|c|}{ Antimicrobial resistance (\%) in Gram negative bacteria } \\
\hline & Study site & $\mathbf{n}$ & AMP & $\mathrm{CAZ}$ & CTX & CRO & FEP & ATM & FOX & AMC & $\mathrm{CN}$ & $S$ & CIP & NA & IPM & RL & $\mathrm{W}$ \\
\hline \multicolumn{18}{|l|}{ E. coli } \\
\hline & Juja & 102 & 50 & 8 & 10 & 12 & 5 & 13 & 24 & 13 & 10 & 15 & 5 & 13 & 0 & 46 & 48 \\
\hline & Slum & 111 & 58 & 9 & 12 & 13 & 7 & 14 & 29 & 15 & 14 & 29 & 9 & 15 & 0 & 52 & 55 \\
\hline \multicolumn{18}{|l|}{$K \cdot$ pneumoniae } \\
\hline & Juja & 88 & 53 & 9 & 11 & 14 & 7 & 15 & 26 & 14 & 13 & 26 & 6 & 24 & 0 & 49 & 50 \\
\hline & Slum & 104 & 60 & 12 & 14 & 14 & 10 & 17 & 32 & 17 & 15 & 33 & 12 & 32 & 0 & 55 & 58 \\
\hline \multicolumn{18}{|l|}{ Salmonella ssp } \\
\hline & Juja & 0 & 0 & 0 & 0 & 0 & 0 & 0 & 0 & 0 & 0 & 0 & 0 & 0 & 0 & 0 & 0 \\
\hline & Slum & 27 & 44 & 7 & 11 & 11 & 4 & 11 & 19 & 15 & 15 & 22 & 4 & 33 & 0 & 48 & 44 \\
\hline \multicolumn{18}{|l|}{ Shigella spp } \\
\hline & Juja & 0 & 0 & 0 & 0 & 0 & 0 & 0 & 0 & 0 & 0 & 0 & 0 & 0 & 0 & 0 & 0 \\
\hline & Slum & 16 & 50 & 6 & 13 & 13 & 0 & 13 & 19 & 13 & 13 & 19 & 6 & 25 & 0 & 44 & 50 \\
\hline \multicolumn{18}{|l|}{ A. baumannii } \\
\hline & Juja & 0 & 0 & 0 & 0 & 0 & 0 & 0 & 0 & 0 & 0 & 0 & 0 & 0 & 0 & 0 & 0 \\
\hline & Slum & 5 & 40 & 20 & 20 & 20 & 0 & 20 & 40 & 20 & 20 & 40 & 20 & 40 & 0 & 40 & 40 \\
\hline \multicolumn{18}{|l|}{$P$. aeruginosa } \\
\hline & Juja & 29 & 52 & 14 & 17 & 21 & 10 & 31 & 41 & 31 & 17 & 41 & 10 & 45 & 0 & 62 & 45 \\
\hline & Slum & 41 & 49 & 15 & 20 & 22 & 12 & 37 & 48 & 37 & 20 & 37 & 15 & 48 & 5 & 44 & 48 \\
\hline \multicolumn{18}{|l|}{ M. morganii } \\
\hline & Juja & 0 & 0 & 0 & 0 & 0 & 0 & 0 & 0 & 0 & 0 & 0 & 0 & 0 & 0 & 0 & 0 \\
\hline & Slum & 15 & 53 & 13 & 13 & 20 & 7 & 20 & 27 & 20 & 13 & 27 & 8 & 33 & 0 & 53 & 47 \\
\hline \multicolumn{18}{|l|}{ S. marcesence } \\
\hline & Juja & 0 & 0 & 0 & 0 & 0 & 0 & 0 & 0 & 0 & 0 & 0 & 0 & 0 & 0 & 0 & 0 \\
\hline & Slum & 5 & 40 & 0 & 20 & 20 & 0 & 20 & 20 & 20 & 0 & 20 & 0 & 20 & 0 & 40 & 20 \\
\hline \multicolumn{18}{|l|}{ Citrobacter spp } \\
\hline & Juja & 49 & 43 & 6 & 8 & 10 & 2 & 12 & 16 & 12 & 8 & 12 & 2 & 16 & 0 & 0 & 0 \\
\hline & Slum & 44 & 43 & 9 & 11 & 11 & 7 & 11 & 18 & 14 & 9 & 18 & 5 & 16 & 0 & 60 & 40 \\
\hline \multicolumn{18}{|l|}{ Enterobacter spp } \\
\hline & Juja & 73 & 51 & 7 & 10 & 11 & 4 & 11 & 14 & 11 & 7 & 15 & 4 & 14 & 0 & 48 & 51 \\
\hline & Slum & 103 & 48 & 10 & 12 & 13 & 5 & 14 & 19 & 13 & 11 & 13 & 5 & 13 & 0 & 50 & 50 \\
\hline \multicolumn{18}{|l|}{ E. tarda } \\
\hline & Slum & 0 & 0 & 0 & 0 & 0 & 0 & 0 & 0 & 0 & 0 & 0 & 0 & 0 & 0 & 0 & 0 \\
\hline & Juja & 10 & 30 & 0 & 0 & 10 & 0 & 10 & 20 & 10 & 0 & 10 & 0 & 20 & 0 & 20 & 10 \\
\hline \multicolumn{18}{|l|}{ Proteus spp } \\
\hline & Slum & 22 & 41 & 10 & 10 & 14 & 5 & 14 & 23 & 23 & 10 & 27 & 5 & 23 & 0 & 45 & 41 \\
\hline & Juja & 44 & 55 & 9 & 11 & 14 & 7 & 16 & 25 & 25 & 14 & 30 & 9 & 30 & 0 & 52 & 50 \\
\hline \multicolumn{18}{|l|}{ H. alvei } \\
\hline & Juja & 0 & 0 & 0 & 0 & 0 & 0 & 0 & 0 & 0 & 0 & 0 & 0 & 0 & 0 & 0 & 0 \\
\hline & Slum & 10 & 40 & 0 & 10 & 10 & 0 & 10 & 10 & 20 & 0 & 20 & 0 & 20 & 0 & 50 & 40 \\
\hline \multicolumn{18}{|l|}{ Yersinia spp } \\
\hline & Juja & 5 & 20 & 0 & 0 & 0 & 0 & 0 & 20 & 0 & 0 & 20 & 0 & 20 & 0 & 20 & 0 \\
\hline & slum & 20 & 25 & 0 & 5 & 5 & 0 & 5 & 10 & 5 & 5 & 15 & 0 & 15 & 0 & 10 & 15 \\
\hline
\end{tabular}


15\%) We also recovered two $P$. aeruginosa isolates from slum sewage that were resistant to imipenem. However, none of the Yersinia species and Edwardisella tarda exhibited these resistance. The resistance prevalence for Klebsiella strains towards any class of antimicrobials was not significantly higher than that of $E$. coli strains (P: 0.36, CI: 1.88, OR: 1.22). Among known Enteropathogens, there was no difference in resistance profiles between Shigella and Salmonella strains (P: 1.25, C1:4.33, OR: 1.25).

\subsection{PCR Detection of $\beta$-Lactamases Gene and Integron in Bacterial Isolates}

A total of 106 selected isolates that were resistant to $3^{\text {rd }}$ generation cephalosporins were screened for carriage of bla $_{\mathrm{TEM}}$, bla $_{\mathrm{CTX}-\mathrm{M}}$, bla $a_{\mathrm{SHV}}$, bla $a_{\mathrm{OXA}}$ and class 1 integron which has widely been reported in Gram-negative bacteria. Carriage of blaTEM $(52 \%)$ was most prevalent ESBL type followed by bla $a_{\text {СтХ-м }}(20 \%)$, bla $a_{S H V}$ $(18 \%)$ while bla $a_{\mathrm{OXA}}(17 \%)$ was the least common, Table 5. All isolates that carried these genes were resistant to ampicillin and at least one $3^{\text {rd }}$ generation cephalosporin (ceftriaxone, cefotaxime and/or ceftazidime). Co-carriage of bla ${ }_{\mathrm{TEM}}$, bla $a_{\mathrm{CTX}-\mathrm{M}}$, bla $a_{\mathrm{OXA}}$ and int 1 was prevalent in $P$. aeruginosa $(13 \%)$ while blas $a_{\mathrm{SHV}}$ was common in Klebsiella species.

\subsection{Fingerprint Analysis}

The phylogeny analysis revealed significant genetic similarity among strains belonging to Escherichia coli, Klebsiella pneumoniae, Enterobacter agglomerans

Table 5. $\beta$-lactamases and integron detected in cephalosporin resistance bacteria.

\begin{tabular}{|c|c|c|c|c|c|c|}
\hline \multirow[t]{2}{*}{ Organism } & \multirow{2}{*}{$\begin{array}{c}\text { Total } \\
\text { screened }\end{array}$} & \multicolumn{5}{|c|}{$\begin{array}{c}\% \text { b-lactamase and integron class } 1 \\
\text { in Gram bacteria isolates. }\end{array}$} \\
\hline & & blaTEM & blaCTX-M & blaSHV & blaOXA & int 1 \\
\hline Citrobacter spp & $\mathrm{n}=10$ & 30 & 10 & 0 & 10 & 0 \\
\hline E. coli & $\mathrm{n}=20$ & 60 & 50 & 15 & 20 & 20 \\
\hline Proteus spp & $\mathrm{n}=9$ & 56 & 22 & 11 & 11 & 11 \\
\hline P.aeruginosa & $\mathrm{n}=15$ & 67 & 47 & 27 & 40 & 47 \\
\hline Salmonella spp & $\mathrm{n}=3$ & 33 & 33 & 0 & 0 & 0 \\
\hline Klebsiella spp & $\mathrm{n}=20$ & 60 & 20 & 50 & 30 & 25 \\
\hline Enterobacter spp & $\mathrm{n}=20$ & 30 & 10 & 10 & 0 & 0 \\
\hline Shigella spp & $\mathrm{n}=2$ & 0 & 0 & 0 & 0 & 0 \\
\hline Acinetobacter baumannii & $\mathrm{n}=2$ & 50 & 0 & 0 & 0 & 50 \\
\hline Morganella morgannii & $\mathrm{n}=2$ & 0 & 0 & 0 & 0 & 0 \\
\hline Serratia marcesence & $\mathrm{n}=1$ & 0 & 0 & 0 & 0 & 0 \\
\hline Edwardisella tarda & $\mathrm{n}=1$ & 0 & 0 & 0 & 0 & 0 \\
\hline Hafnia alvei & $\mathrm{n}=1$ & 0 & 0 & 0 & 0 & 0 \\
\hline
\end{tabular}


and Proteus mirabilis strains but less relatedness was noted among strains belonging to Citrobacter freundii, Figures 3-7. Further analysis showed possible clonal expansion within the environmental ecosystems of $E$. agglomerans Figure 4, cluster 2), Klebsiella pneumoniae (Figure 5, cluster 2) and E. coli (Figure 8, cluster 1,3$)$. Such strains had identical resistance phenotypes and shared more than $80 \%$ genetic similarity. Other strains such as Salmonella Typhi showed a similarity of above $70 \%$ in terms of resistance patterns but differed significantly

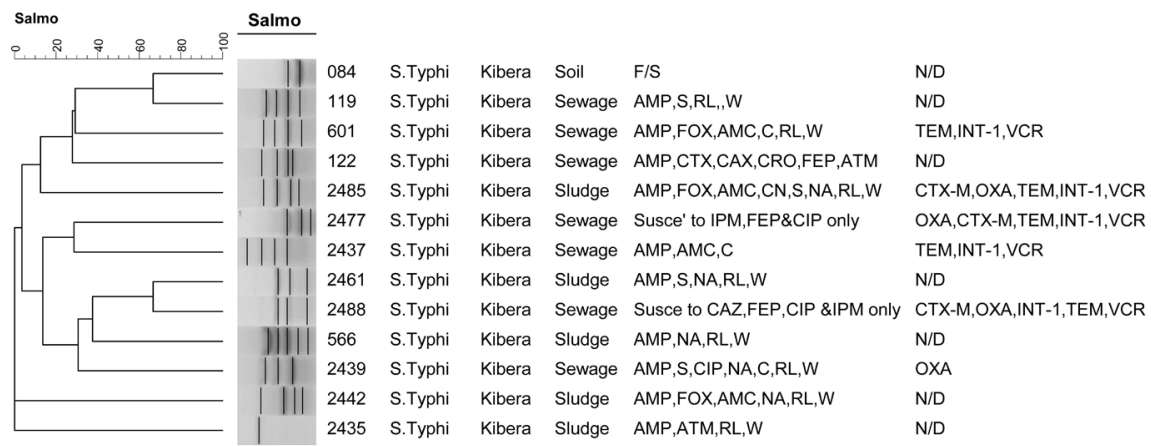

Figure 3. Cluster analysis of recover Salmonellatyphi from sewage, soil and sludge. The figure show phylogeny analysis of Salmonella Typhi isolates from sewage sludge and soil sample from Kibera slums. Description of various acronyms on this figure is as follows; $S$. Typhi: Salmonella Typhi, TEM: Temoneria $\beta$-lactamase, CTX-M: Cefotaxime Munich $\beta$-lactamase, SHV: Sulhydryl variant $\beta$-lactamase, Int-1: Integron, VCR: Variable cassette region, N/D: none of the screened genes were detected.

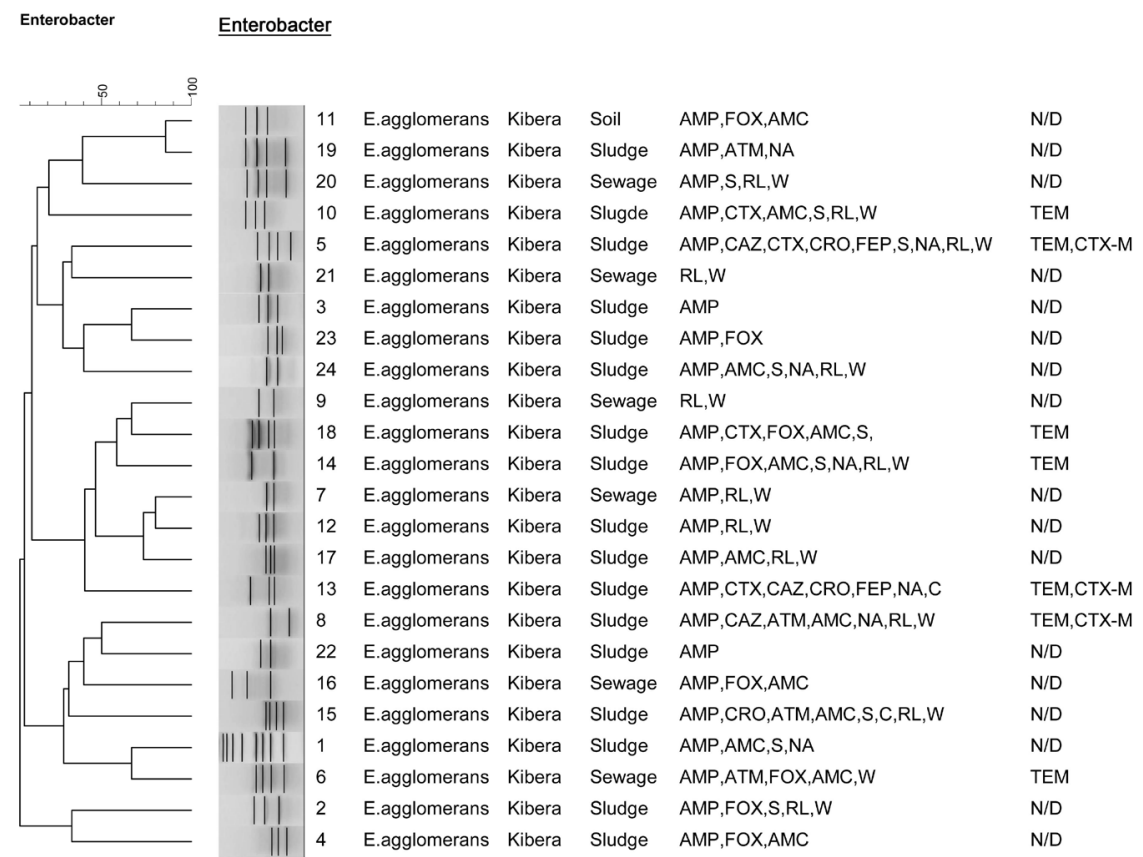

Figure 4. Cluster analysis of recover Enterobacter agglomerans from sewage, soil and sludge. The figure show phylogeny analysis of Enterobacter agglomerans isolates from sewage sludge and soil sample from Kibera slums. Description of various acronyms on this figure is as follows; E. agglomerans. Enterobacter agglomerans, N/D: none of the screened genes were detected. 


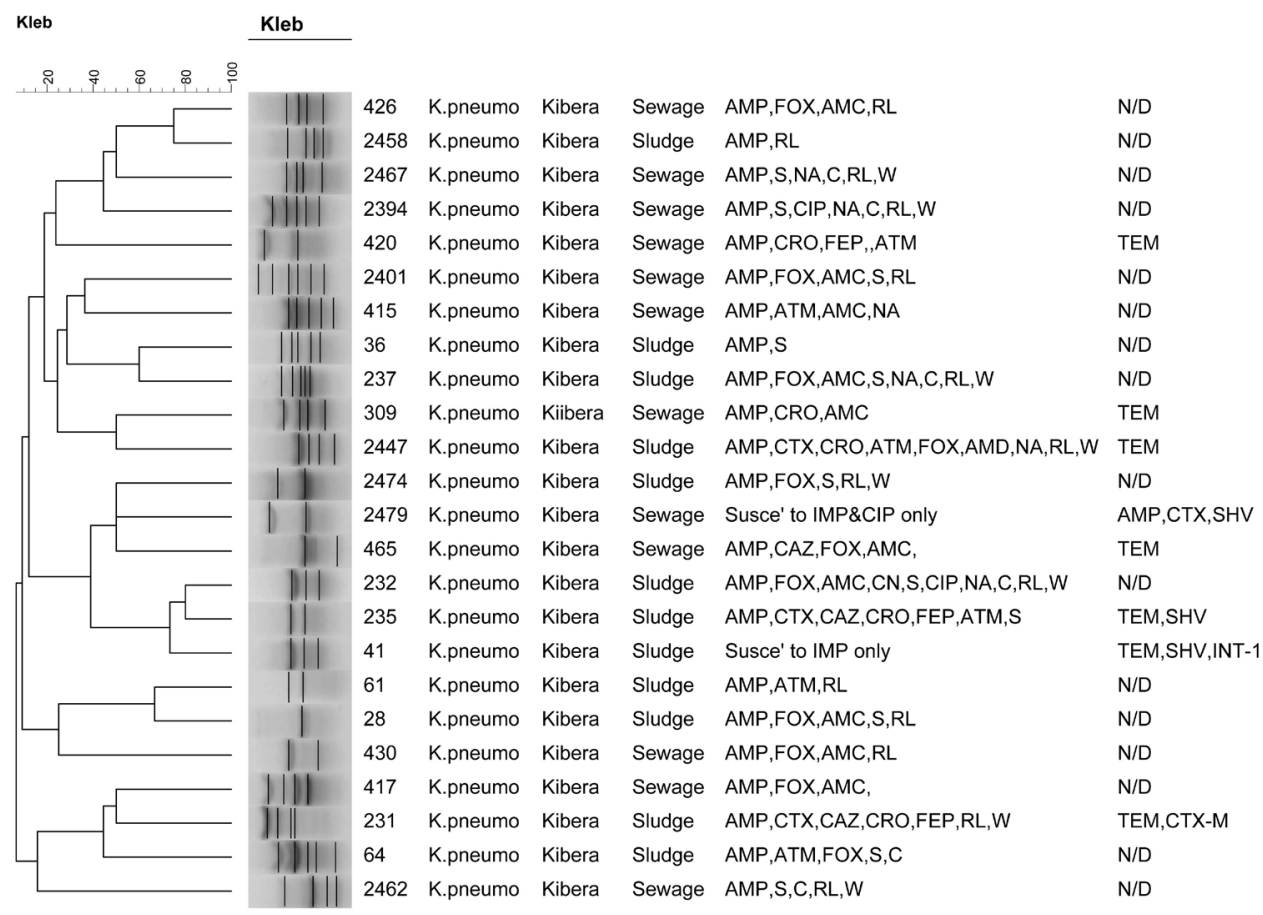

Figure 5. Cluster analysis of recover Klebsiella pneumoniae from sewage and sludge. The figure show phylogeny analysis of Klebsiella pneumoniae isolates from sewage sludge and soil sample from Kibera slums. Description of various acronyms on this figure is as follows; K pneumo: Klebsiella pneumoniae, Int-1: Integron, N/D: none of the targeted gens was detected.

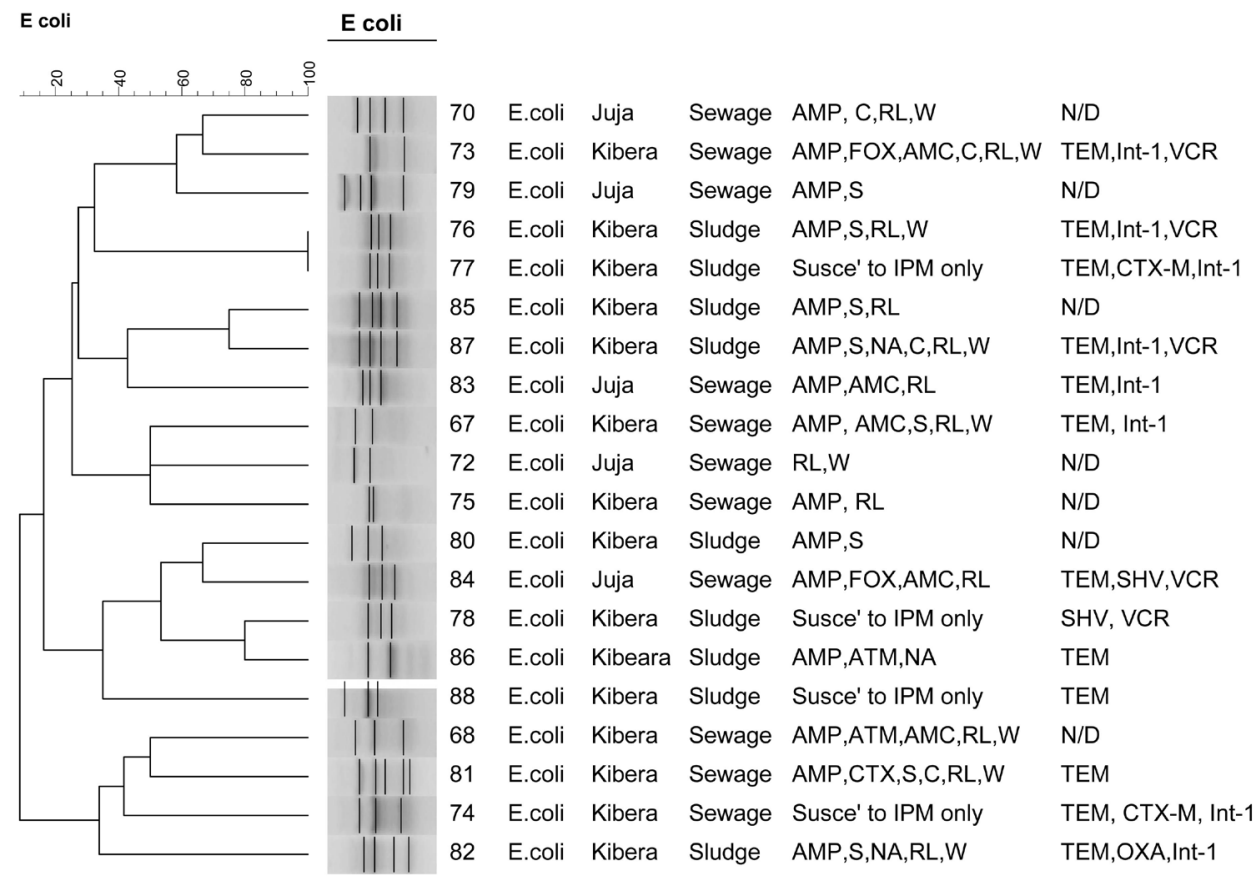

Figure 6. Cluster analysis of recover Escherichia coli from sewage and sludge. The figure show phylogeny analysis of Escherichia coli isolates from sewage sludge and soil sample from Kibera slum and Juja town. Description of various acronyms on on this figure is as follows; E.coli: Escherichia coli, Int-1: Integron, VCR: Variable cassette region, So: Soil, Se: sewage, Sl: Sludge, N/D: none of the targeted gens was detected. 


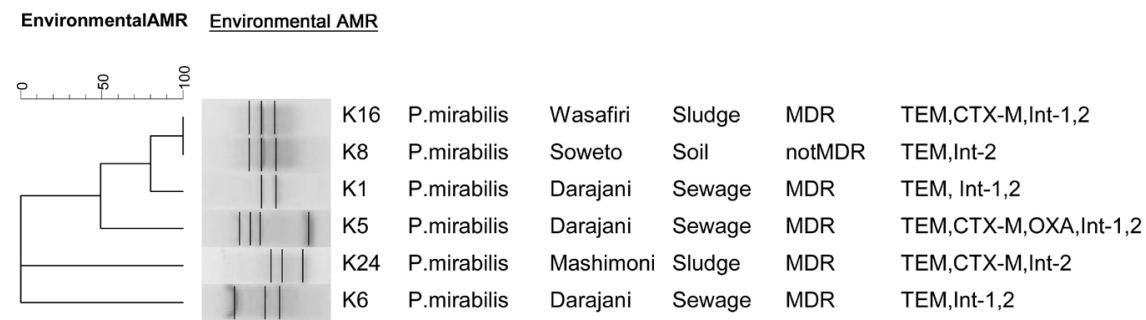

Figure 7. Cluster analysis of recover Proteus mirabilis from soil, sludge and sewage samples. The figure show phylogeny analysis of Proteus mirabilis isolates from sewage sludge and soil sample from Kibera slums. Description of various acronyms on this figure is as follows; P. mirabilis: Proteus mirabilis, MDR: Multiple drug resistance, TEM: Temoneria $\beta$-lactamase, CTX-M: Cefotaxime Munich $\beta$-lactamase, SHV: Sulhydryl variant $\beta$-lactamase, Int: Integron, VCR: Variable cassette region.

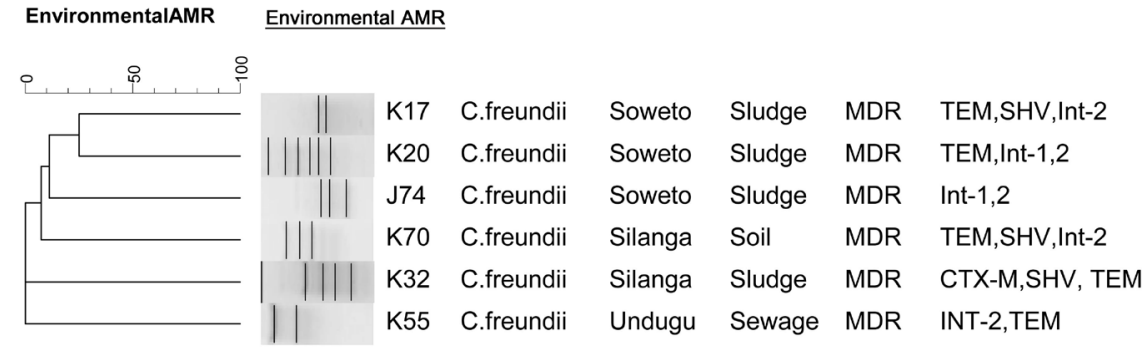

Figure 8. Cluster analysis of recover Citrobacter freundii from sewage, soil and sludge. The figure show phylogeny analysis of Citrobacter freundii isolates from sewage sludge and soil sample from Kibera slums. Description of various acronyms on this figure is as follows; $C$. freundii: Citrobacter freundii, Int: Integron, N/D: none of the targeted genes were detected.

based on the banding patterns further indicating that different clones may have acquired similar sets of resistance determinants independent (Figure 3). Tight clustering of E. coli strains from slum (Kibera) and the middle class settlement (Juja town) was also noted; these isolates however had different resistance phenotypes suggesting independent evolution (Figure 8, cluster 1). Apparently, sample type and study site was not a key determinant in isolates clustering. These results call for application of high resolution typing of such strains using whole genome analysis strategies in future studies in order to identify the most stable clones that could significantly be related to emergence and spread of AMR.

\section{Discussion}

It is estimated that by the year 2050 the urban population will double. However, with poor urban planning especially in developing countries, high unemployment rate and poverty, informal settlements are increasingly becoming rampant [15]. Densely populated neighborhoods with poor sanitation infrastructure pose a serious health risk [16]. Previous independent observations have noted that raw and partially processed excreta leaks from domestic toilets in Kibera slums and find their way in the open sewer [17]. In order to reduce chances of release 
of enteric bacteria and antimicrobial residues into the environment, there is a need for provision of improved basic sanitation such as clean toilets and proper drainage system in slums and urban centers.

In the current study we recorded a diverse group of non-fastidious Gram-negative bacteria isolates in environmental samples from slum and middleclass setting. Majority of genera isolated are those known to reside in human and animal gut such as Escherichia, Klebsiella and some are known Enteropathogens such as Salmonella and Shigella species. Although clinical reports of Salmonella and Shigella species is on declines, environment colonization still remain a possible source of typhoid, and Shigellosis pathogens. It is likely that human fecal and untreated domestic waste could be seeding bacteria of enteric origin into the environment and some of these may have clinical origins based on their resistance profiles. Our study underpins the role of environmental contamination as a driving factor for emergence of outbreaks some of which are caused by MDR strains. We also isolated MDR $P$. aeruginosa and $A$. baumannii; these organisms have previously been associated with wide range of difficult-to-treat infections [18]. The bacteria diversity recorded in this study is comparatively broader compared to those reported in a related study conducted in 2013 and analyzed the microbial content and resistance profiles of isolates obtained from the sewage-contaminated Nairobi river [19]. There is therefore a clear indication that ecosystem contamination with domestic wastes is on a gradual increase in contamination of the environment.

High antimicrobial resistances towards ampicillin (57\%), trimethoprim (56\%), cefoxitin (28\%), sulfamethoxazole (27\%) and nalidixic acid (26\%) was recorded in isolates from slum. These findings strongly suggest that bacteria from the environment compartments are able accumulate many antimicrobial resistance determinants. The occurrence of such strains with resistance to a combination of antimicrobials raises concerns because if such strains are implicated in infections, available treatment options would be highly limited.

Our data show a higher prevalence of resistance to antimicrobials among isolates recovered from slum environment compartments compared to a previous study conducted in the same slum, Kibera in 2012 [20]. The former study that sought to characterize E. coli, Salmonella and Shigella species in water, soil, vegetables and meat reported and reported a resistance prevalence of $56.8 \%$ towards ampicillin against $57 \%$ reported here, $13.6 \%$ for streptomycin against $27 \%$, $4.9 \%$ for nalidixic acid against $26 \%$ and $2.5 \%$ for gentamicin against $14 \%$ recorded in our study. Thus, the values from this previous study were generally lower than what we reported in the present study by a factor of greater than $10 \%$. Although the reasons behind this apparent rise in resistance prevalence is not clear, previous study have reported that more than $80 \%$ of antibiotics consumed by humans and animals are excreted through urine and feces [21]. Therefore, a significant amount of active residues may end up in the sewage, sludge and other environment compartments thereby presenting a strong selection pressure that favor proliferation of MDR strains. Contamination of soil with enteric bacteria 
and antimicrobial compounds comes from domestic and municipal waste and also from sewage sludge systems [22]. Significant amounts of antimicrobial residues belonging to more than 6 antimicrobial classes have previously been reported in sewage in Asia and Europe and some classes of antimicrobials such as tetracycline and chloramphenicol can persist in soil, sewage and sludge for decades [23]. The presence of such agents in the environment can consequently provide a strong selective pressure that preferentially allows MDR clones to spread [24]. Although our study did not assess presence of antimicrobial agents residues in the environment, our finds strongly underpins the need to such a study.

Higher levels of antimicrobial resistances than reported in our study with greater than $30 \%$ towards ceftazidime, cefotaxime, ceftriaxone, ciprofloxacin, aztreonam and gentamicin in $A$. baumannii, E. coli, $P$. aeruginosa and $K$. pneumoniae from blood, tracheal aspirates, wound, pus and urine samples have been documented in Kenya [25] [26]. Our study however show an increase in antimicrobial resistance in environmental compartments compared to a previous study conducted in Kakamega town in Kenya [27]. The former study reported E. coli from recovered from dumpsites, sludge and wastewater. We reported more 3\% increase in resistance towards amoxicillin-clavulanic acid, gentamicin and ciprofloxacin in Citrobacter species, Enterobacter spp. and Klebsiella spp. compared to former study. Although the threat of antimicrobial resistance is more profound in clinical settings, our findings show environmental bacteria strains are increasingly becoming resistant and therefore should not be overlooked.

Carriage of bla $a_{\mathrm{TEM}}(52 \%)$ was most prevalent in all bacteria species followed by bla $_{\text {Стх-м }}(20 \%)$, bla $a_{S H V}(18 \%)$ while bla OXA $_{\text {O }}(17 \%)$ was the least common bla gene. Co-carriage of bla $a_{\text {TЕM }}, b l a_{\text {СТХ-M }}$, bla $a_{\text {OXA }}$ and int 1 was prevalent in $P$. aeruginosa (13\%) while blas $a_{\mathrm{SV}}$ was common in Klebsiella species. Previous studies have shown that most bacteria that carry bla genes also carry integrons which are mostly harbored in mobile genetic elements such plasmids therefore suggesting spread and acquisition in bacteria community [28].

Most of our isolates had less than $70 \%$ similarity with distinct resistance phenotypes and genotypes suggesting very little evidence of clonal expansion in the environment. Tight clustering of $\geq 70$ similarity matrix among some few Escherichia coli, Klebsiella pneumoniae, Enterobacter agglomerans and Proteus mirabilis strains isolate however suggests independent acquisition of a similar set of resistance determinants among isolates with different profiles. Although we were not able to determine the cause of above mentioned resistance features, such phenomenon has previously been associated with carriage of genetic elements such as plasmids [29].

\section{Conclusion}

The high diversity of multiple-drugs resistance in sewage and sludge samples observed in this study pose a serious public health hazard in emanation of seri- 
ous infection. Unregulated discharge of domestic waste highly polluted with fecal material on open sewer is a great risk to many residents of over-polluted Kibera informal settlements. There is therefore a dire need for proper sewage management to reduce or elimine the serious health risk posed by open sewer.

\section{Study Limitations}

Our study experienced a number of shortcomings that can be a basis for formulation of stronger studies in futures.

1) The broad range of bacteria genera reported in this study makes focused discussion challenging.

2) We were only able to screen for a few $\beta$-lactamases genes. Our study was also not able to determine the bases of resistance to other classes of antimicrobial agents such as aminoglycosides and fluoroquinolones.

3) We were unable to determine the content of detected int 1 and whether they were born in mobile genetic elements such as plasmids.

4) Low resolution $(G T G)^{5}$ fingerprint method was used to establish bacteria phylogeny and genetic relatedness. Futures studies should apply high resolution methods such as whole genome sequencing and SNP typing which can shed more light resistance gene content and bacteria evolution in the environmental compartments.

\section{Acknowledgements}

The authors wish to send special thanks to KEMRI, CMR for allowing us to conduct this research study at the institution. We are also grateful to the CMR molecular biology laboratory staff for their relentless support and making this study a success. This work was partially funded by the Kenya National Research Fund (NRF).

\section{Author Contributions}

John Maina designed and wrote the research protocol for this study in addition to conducting lab work and the manuscript writing. Helen Onyango assisted in lab work and statistical analysis. John Kiiru assisted in designing the study and writing the manuscript. All authors read and approved final manuscript. Anne Muigai and Perpetual Ndung'u provided assist in protocol correction and amendments in study methodologies. Joel Mukaya, Susan Wambui, Terry Judah, Joyce Kinyua, Joystella Muriuki, Lynne Chesenge, Lydia Kisoo, Rebecca Thuku, Boniface Wachira, Vincent Bett and Thomas Gachuki offered laboratory technical support.

\section{Authors' Information}

John Maina is a student of Medical Microbiology at the institute of tropical medicine and infectious diseases (ITROMID), JKUAT. John Kiiru is a principal researcher at Centre for Microbiology, KEMRI. Hellen Onyango and Perpetual 
Ndung'u are lectures at JKUAT while Anne Muigai is a professor of genetics in the same university. Joel Mukaya and Thomas Gachuki are Masters Students at ITROMID, JKUAT. Joyce Kinyua, Joystella Muriuki, Lynne Chesenge, Lydia Kisoo, Rebecca Thuku and Boniface Wachira are Biotechnology students in JKUAT. Susan Wambui, Terry Judah and Vincent Bett are assistant researchers in KEMRI.

\section{Conflict of Interest}

The authors declare no conflicts of interest regarding the publication of this paper.

\section{References}

[1] O’Neill, J. (2016) Tackling Drug-Resistant Infections Globally: Final Report and Recommendations. http://amr-review.org/sites/default/files/160525 Final\%20paper with\%20cover.pdf

[2] Picao, R.C., Cardoso, J.P., Campana, E.H., Nicoletti, A.G., Petrolini, F.V., Assis, D.M., et al. (2013) The Route of Antimicrobial Resistance from the Hospital Effluent to the Environment: Focus on the Occurrence of KPC-Producing Aeromonas spp. and Enterobacteriaceae in Sewage. Diagnostic Microbiology and Infectious Disease, 76, 80-85. https://doi.org/10.1016/j.diagmicrobio.2013.02.001

[3] Fresia, P., Antelo, V., Salazar, C., Gimenez, M., D’Alessandro, B., Afshinnekoo, E., et al. (2019) Urban Metagenomics Uncover Antibiotic Resistance Reservoirs in Coastal Beach and Sewage Waters. Microbiome, 7, 35. https://doi.org/10.1186/s40168-019-0648-Z

[4] Novo, A., Andre, S., Viana, P., Nunes, O.C. and Manaia, C.M. (2013) Antibiotic Resistance, Antimicrobial Residues and Bacterial Community Composition in Urban Wastewater. Water Research, 47, 1875-1887. https://doi.org/10.1016/j.watres.2013.01.010

[5] White, P.A., McIver, C.J. and Rawlinson, W.D. (2001) Integrons and Gene Cassettes in the Enterobacteriaceae. Antimicrobial Agents and Chemotherapy, 45, 2658-2661. https://doi.org/10.1128/AAC.45.9.2658-2661.2001

[6] Sarmah, A.K., Meyer, M.T. and Boxall, A.B. (2006) A Global Perspective on the Use, Sales, Exposure Pathways, Occurrence, Fate and Effects of Veterinary Antibiotics (VAs) in the Environment. Chemosphere, 65, 725-759.

https://doi.org/10.1016/j.chemosphere.2006.03.026

[7] Wellington, E.M., Boxall, A.B., Cross, P., Feil, E.J., Gaze, W.H., Hawkey, P.M., et al. (2013) The Role of the Natural Environment in the Emergence of Antibiotic Resistance in Gram-Negative Bacteria. The Lancet Infectious Diseases, 13, 155-165. https://doi.org/10.1016/S1473-3099(12)70317-1

[8] Cleary, D.W., Bishop, A.H., Zhang, L., Topp, E., Wellington, E.M. and Gaze, W.H. (2016) Long-Term Antibiotic Exposure in Soil Is Associated with Changes in Microbial Community Structure and Prevalence of Class 1 Integrons. FEMS Microbiology Ecology, 92, fiw159. https://doi.org/10.1093/femsec/fiw159

[9] Okeke, I.N., Aboderin, O.A., Byarugaba, D.K., Ojo, K.K. and Opintan, J.A. (2007) Growing Problem of Multidrug-Resistant Enteric Pathogens in Africa. Emerging Infectious Diseases, 13, 1640-1646. https://doi.org/10.3201/eid1311.070674

[10] Yong, D.C., Thompson, J.S. and Prytula, A. (1985) Rapid Microbiochemical Method 
for Presumptive Identification of Gastroenteritis-Associated Members of the Family Enterobacteriaceae. Journal of Clinical Microbiology, 21, 914-918.

[11] El-Sokkary, M.M.A. and Abdelmegeed, E.S. (2015) Characterisation of Class 1 Integron among Escherichia coli Isolated from Mansoura University Hospitals in Egypt. Advances in Microbiology, 5, 269-277. https://doi.org/10.4236/aim.2015.54025

[12] Hasman, H., Mevius, D., Veldman, K., Olesen, I. and Aarestrup, F.M. (2005) $\beta$-Lactamases among Extended-Spectrum $\beta$-Lactamase (ESBL)-Resistant Salmonella from Poultry, Poultry Products and Human Patients in The Netherlands. Journal of Antimicrobial Chemotherapy, 56, 115-121. https://doi.org/10.1093/jac/dki190

[13] Mohapatra, B.R., Broersma, K. and Mazumder, A. (2007) Comparison of Five Rep-PCR Genomic Fingerprinting Methods for Differentiation of Fecal Escherichia coli from Humans, Poultry and Wild Birds. FEMS Microbiology Letters, 277, 98-106. https://doi.org/10.1111/j.1574-6968.2007.00948.x

[14] Vaez, H., Moghim, S., Nasr, E.B. and Ghasemian, S.H. (2015) Clonal Relatedness among Imipenem-Resistant Pseudomonas aeruginosa Isolated from ICU-Hospitalized Patients. Critical Care Research and Practice, 2015, Article ID: 983207. https://doi.org/10.1155/2015/983207

[15] Alirol, E., Getaz, L., Stoll, B., Chappuis, F. and Loutan, L. (2011) Urbanisation and Infectious Diseases in a Globalised World. The Lancet Infectious Diseases, 11, 131-141. https://doi.org/10.1016/S1473-3099(10)70223-1

[16] Hutton, G. and Chase, C. (2017) Water Supply, Sanitation, and Hygiene. In: Disease Control Priorities, 3rd Edition (Volume 7): Injury Prevention and Environmental Health, The World Bank, Washington DC, 171-198. https://doi.org/10.1596/978-1-4648-0522-6 ch9

[17] Akullian, A., Ng'eno, E., Matheson, A.I., Cosmas, L., Macharia, D., Fields, B., et al. (2015) Environmental Transmission of Typhoid Fever in an Urban Slum. PLOS Neglected Tropical Diseases, 9, e0004212. https://doi.org/10.1371/journal.pntd.0004212

[18] Bonomo, R.A. and Szabo, D. (2006) Mechanisms of Multidrug Resistance in Acinetobacter Species and Pseudomonas aeruginosa. Clinical Infectious Diseases, 43, S49-S56. https://doi.org/10.1086/504477

[19] Musyoki, A.M., Suleiman, M.A., Mbithi, J.N. and John, M. (2013) Water-Borne Bacterial Pathogens in Surface Waters of Nairobi River and Health Implication to Communities Downstream Athi River. Environmental Microbiology, 3, 4-10.

[20] Christabel, M., Budambula, N., Kiiru, J. and Kariuki, S. (2012) Characterization of Antibiotic Resistance in Environmental Enteric Pathogens from Kibera Slum in Nairobi-Kenya. African Journal of Bacteriology Research, 4, 46-54. https://doi.org/10.5897/JBR12.008

[21] Kummerer, K. (2009) Antibiotics in the Aquatic Environment-A Review-Part I. Chemosphere, 75, 417-434. https://doi.org/10.1016/j.chemosphere.2008.11.086

[22] Manaia, C.M., Macedo, G., Fatta-Kassinos, D. and Nunes, O.C. (2016) Antibiotic Resistance in Urban Aquatic Environments: Can It Be Controlled? Applied Microbiology and Biotechnology, 100, 1543-1557. https://doi.org/10.1007/s00253-015-7202-0

[23] Yan, Q., Gao, X., Chen, Y.-P., Peng, X.-Y., Zhang, Y.-X., Gan, X.-M., et al. (2014) Occurrence, Fate and Ecotoxicological Assessment of Pharmaceutically Active Compounds in Wastewater and Sludge from Wastewater Treatment Plants in Chongqing, the Three Gorges Reservoir Area. Science of the Total Environment, 470-471, 618-630. https://doi.org/10.1016/j.scitotenv.2013.09.032 
[24] Sarmah, A.K., Meyer, M.T. and Boxall, A.B. (2006) A Global Perspective on the Use, Sales, Exposure Pathways, Occurrence, Fate and Effects of Veterinary Antibiotics (VAs) in the Environment. Chemosphere, 65, 725-759. https://doi.org/10.1016/j.chemosphere.2006.03.026

[25] Onyango, H.A., Ngugi, C., Maina, J. and Kiiru, J. (2018) Urinary Tract Infection among Pregnant Women at Pumwani Maternity Hospital, Nairobi, Kenya: Bacterial Etiologic Agents, Antimicrobial Susceptibility Profiles and Associated Risk Factors. Advances in Microbiology, 8, 175-187. https://doi.org/10.4236/aim.2018.83012

[26] Kilivwa, Mukaya, J.S., Maina, J., Museve, B., Nyerere, A.K. and Kiiru, J. (2018) Antimicrobial Resistance Profile and Genetic Profiling of Pseudomonas aeruginosa Strains Obtained from Different Inpatient Wards at Kenyatta National Hospital. IOSR Journal of Pharmacy and Biological Sciences, 13, 1-9.

[27] Malaho, C., Wawire, S.A. and Shivoga, W.A. (2018) Antimicrobial Resistance Patterns of Enterobacteriaceae Recovered from Wastewater, Sludge and Dumpsite Environments in Kakamega Town, Kenya. African Journal of Microbiology Research, 12, 673-680.

[28] Spanu, T., Luzzaro, F., Perilli, M., Amicosante, G., Toniolo, A. and Fadda, G. (2002) Occurrence of Extended-Spectrum Beta-Lactamases in Members of the Family Enterobacteriaceae in Italy: Implications for Resistance to $\beta$-Lactams and Other Antimicrobial Drugs. Antimicrobial Agents and Chemotherapy, 46, 196-202.

https://doi.org/10.1128/AAC.46.1.196-202.2002

[29] Vounba, P., Arsenault, J., Bada-Alambedji, R. and Fairbrother, J.M. (2019) Pathogenic Potential and the Role of Clones and Plasmids in $\beta$-Lactamase-Producing $E$. coli from Chicken Faeces in Vietnam. BMC Veterinary Research, 15, 106. https://doi.org/10.1186/s12917-019-1849-1 